\title{
Risk assessment for pharmaceutical products in the environment
}

\author{
V. Popov ${ }^{1}$, V. Tomenko ${ }^{1}$, C. A. Brebbia ${ }^{1}$, A. H. Piersma ${ }^{2}$ \\ \& M. Luijten ${ }^{2}$ \\ ${ }^{I}$ Wessex Institute of Technology, Environmental and Fluid Mechanics, \\ $U K$ \\ ${ }^{2}$ Laboratory for Toxicology, Pathology and Genetics, RIVM, \\ The Netherlands
}

\begin{abstract}
Recently, a Specific Targeted Research Project (STREP), termed F\&F, supported by the European Commission under the Sixth Framework Programme Priority 5 on Food Quality and Safety (Contract No. FOOD-CT-2004-513953, http://foodandfecundity.factlink.net) on "Pharmaceutical products in the environment: Development and employment of novel methods for assessing their origin, fate and effects on human fecundity" has been established. The F\&F project intends to integrate research groups from different disciplines (risk assessors managers, clinical epidemiologists, endocrinologist, biochemists, as well as experts in biochemical and chemical diagnostics) for a better understanding of the extent of the problem. Our part in the project focuses on evaluating the risk assessment of PPs in food and in the environment.

This work will pinpoint study and summarize the RA of different PPs, their origin and their influence on fecundity.
\end{abstract}

\section{Risk assessment for PPs in the environment}

The risk assessment part of the F\&F project includes:

1. Identification of a platform of endocrine disrupting substances in the food on a regional basis within Europe;

2. Identification and assessment of other risk factors that may influence fecundity, including age, reproductive history, diet and nutrition, socioeconomic status, habits and lifestyle; 
3. Survey of national/regional fecundity in European populations;

4. Survey of dietary habits across the selected regions;

5. Contribution towards the development of a suite of biomarkers and high throughput screening assays, suitable for potential applications in mechanistic research and human medical and epidemiological applications;

6. Qualitative and, potentially, quantitative assessment of the risk posed to human fecundity through exposure to chemicals in food.

The main elements of the risk assessment task of the project are shown in Figure 1. It can be seen that the first step consists of Identification of potential PPs with an effect on human fecundity. Once the PPs are selected required data for Exposure Assessment as well as Dose Response curves are collected through corresponding project tasks, which is finally used for the Risk Characterization and definition of Risk Management Strategies.

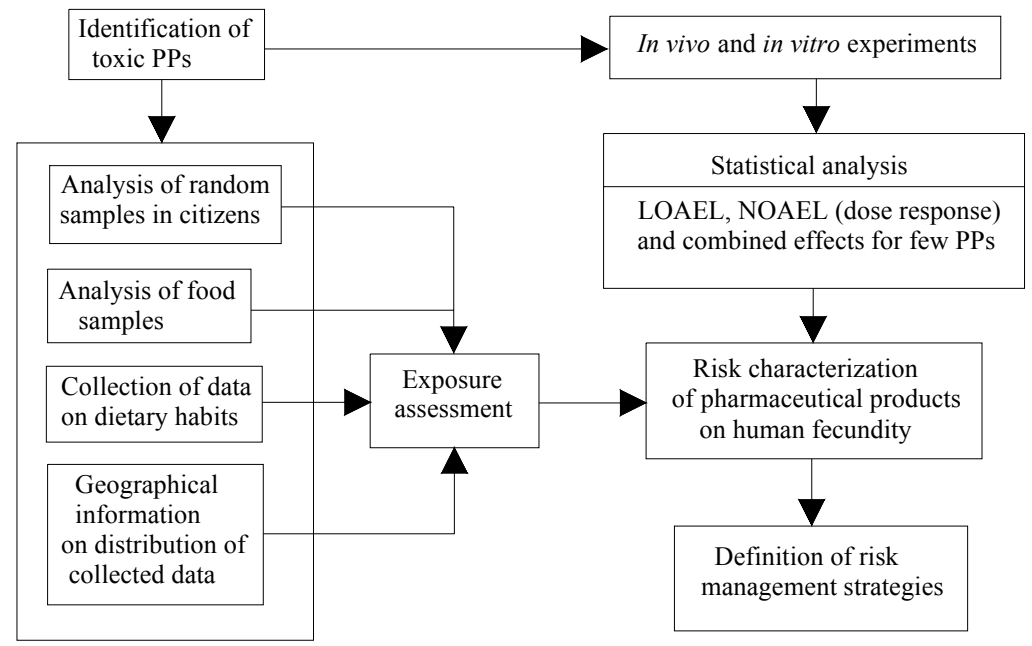

Figure 1: $\quad$ Risk assessment strategy for the F\&F project.

\subsection{Selection of PPs}

After the first six months of the project three compounds were selected that were considered relevant within the framework of F\&F, though this number will be increased to twenty by the end of the project. A prioritization list of pharmaceutical products (PP) bearing a high potential of affecting human fecundity by entering the food chain has been created, based on an extensive literature search, and by considering the following issues during the selection process: (i) Does the available data indicate there is evidence that the chemical causes endocrine disrupting effects related to fecundity? (ii) Is the production volume and/or use of the PP sufficiently large to cause concern? (iii) Is the PP sufficiently persistent in the environment? (iv) Can a clear exposure route be identified by which the PP would enter the food chain? 
The main problem at this stage is that there is lack of data necessary to answer the above questions for all of the PPs nowadays in use. Several chemicals have been selected for which data exists indicating positive answers to all or several of the questions (i) to (iv). In the first stage the selection process was focused on chemicals for which evidence exists for positive answer to (i). Those chemicals selected in the first stage were further investigated for additional information on the other three questions. The chemicals with more positive answers to questions (i) to (iv) and with more evidence from previous research to support those findings are of more importance to the study.

The identified compounds were ethynylestradiol, medroxyprogesterone acetate, and diethylstilbestrol.

Some of the available data on EE2 in respect to the biological effectivity, production volumes, exposures and environmental fate is given below. Similar data was gathered for the other two compounds but can be found elsewhere [1].

\subsubsection{7 $\alpha$-Ethynylestradiol (EE2)}

EE2 is used in oral contraceptives in humans. The effect of EE2 on animals has been investigated in several studies and some results are given below. These studies may give some indication of what the no observed effect concentration (NOEC) and lowest observed effect concentration (LOEC) in humans might be.

The synthetic oestrogen hormone, $17 \alpha$-ethinyloestradiol can be used in human medicine to treat various gynaecological disorders and post-menopausal breast cancer. However, its largest use is in oral contraceptives, when it is usually administered in combination with a synthetic progestin. Its concentration in the contraceptive pill ranges from 20 to $50 \mu \mathrm{g}$, with $35 \mu \mathrm{g}$ most commonly prescribed [2]. An annual use of 0.029 tonnes of $17 \alpha$-ethinyloestradiol has been estimated in the UK [3]. By comparison, it has been estimated that 0.088 tonnes of oral contraceptives (17 $\alpha$-ethinyloestradiol and mestranol) are used annually in the USA [4].

Synthetic estrogens (EE2 and mestranol) are more resistant to microbial degradation than natural steroids (estradiol, estrone, estriol). The data on the physico-chemical properties of EE2 and its environmental fate indicate that the compound is relatively persistent in the aquatic environment. It is likely that adsorption of EE2 to soil is a major removal process ( $\log \mathrm{Koc}=3.8$ ).

There are several routes that may lead to contamination of food or water with EE2, see Figure 2. If we neglect routes related to effects which would exist just in the vicinity of the production plant and should be negligible if the necessary precautions are in operation at the plant, then the most likely route would be through the human usage, where EE2 would either be discarded and end up in landfills or would end up in sewage through human excreta and from there would enter a sewage treatment plant (STP).

EE2 can be further transported from a landfill as effluent through the landfill effluent treatment system, and from there into the sanitary sewage or STP, or could be released into surface waters or land, depending on the level of treatment. Landfill leachate can percolate the containment system and pollute 
soil and groundwater, however, this route should not represent a significant treat to the environment in a well designed and maintained landfill and therefore will not considered further.

The sewage sludge from a STP, among other options like incineration for example, may be disposed into a landfill, or could be used in agriculture. From the agricultural fields the EDs compounds may be transported to surface waters, soil and groundwater by leaching, dissipation and run-off. If EE2 is transported to surface waters it may end up in the food chain by bioacumultaion in fish or as water for domestic use, and if it reaches groundwater it may further be used as tap water for human consumption. Once sewage sludge is applied to agricultural fields EE2 may end up in plants through plant uptake.

Unless direct measurements show otherwise, the risks from EE2 being present in the air due to evaporation from landfills, sewage treatment plants and agricultural fields where sewage sludge is applied, are considered to be negligible.

There is awareness of the importance of investigating the presence of EE2 in the environment, and many of the previous studies included EE2 as the main representative of the synthetic steroids.

Evidence exists of presence of EE2 in the environment. EE2 was found basically in every media where an attempt was made for its detection, i.e., raw sewage [5], STP effluent [6], rivers [7] and even groundwater [8]. The detection of EE2 is difficult since its concentrations are usually on the detection limit, but this certainly does not mean that the risk from EE2 is negligible. EE2 can cause changes in animals in very low concentrations. Chronic exposure under laboratory conditions, including studies of chronic exposure over two complete generations, to as little as $0.6 \mathrm{ng} / \mathrm{l} \mathrm{EE} 2$ (below the limits of chemical detection for most effluents) was sufficient to sex reverse male fish (primarily zebrafish, with some work on the stickleback) and $1.5 \mathrm{ng} / \mathrm{l}$ stimulated vitellogenesis in juvenile fish (COMPREHEND 2002). Bioaccumulation can increase the EE2 concentrations by several orders of magnitude [6]. One should also not forget that EE2 concentrations in the STPs could be increased by the partial conversion of other drugs into this molecule [9]. Finally, several studies have shown that EE2 is more persistent in the environment than the natural estrogens $[5,10,11]$.

\subsection{Food to be tested}

Since there is a possibility that banned growth promoting hormones may still be in use, obvious choice of food to be tested is: beef, veal, pork, poultry and fish. Food processing steps, such as cooking, smoking and fermenting, appear to have little effect on the steroid patterns.

Steroid hormones pass the blood-milk barrier. A strong correlation of the progesterone, pregnenolone, androstenedione and estrone levels with the milk fat content have been proved. The levels are higher by factor 10 to 20 in butter than in milk because of the fat content. Hartmann et al. [12] reported that the main source of natural estrogens and progesterone are dairy products (60-80\%). A similar pattern can be expected for synthetic steroids, should they end up in the 
food of animals. Food processing does not seem to influence the amounts and ratios of the investigated hormones.

Eggs are a considerable source of hormonally active natural steroids and their precursors and therefore they should be included in the list of food to be tested for synthetic steroids, since any increase in hormone levels would naturally appear in eggs as well.



Figure 2: $\quad$ Exposure routes for EE2.

The presence of $17 \beta$-estradiol and estrone in French beans (Phaseolus vulgaris) has been proved, with gas chromatography-mass spectrometry. It seems possible that some synthetic steroids end-up in plants through uptake from the soil, after it has been treated with sewage sludge.

Finally, baby food must be tested since children are especially vulnerable to endocrine disruptors.

\subsection{Endpoints in the environment to be tested}

\subsubsection{Wastewater, surface water, tap water}

EE2, E2 and other estrogenic compounds have regularly been detected in STPs. In several cases these compounds have been detected in surface waters as well. Finally, it is possible that they end up in the domestic water system, but the question is: in what concentrations? Water supply systems which rely mainly on 
rivers, like for example the water supply system of London, where $74 \%$ of the water comes from the River Thames, $15 \%$ from River Lee and 11\% from groundwater, may be especially vulnerable.

\subsubsection{Sewage sludge and treated soil}

Sewage sludge, which is used in agriculture, will be tested in several STP for presence of selected compounds and if contaminated the crops on the treated fields with the sewage sludge will be tested and closely followed for any traces of the selected PPs.

\section{Mathematical methods which will be considered for the RA}

\subsection{Threshold methods}

It is assumed that there is a threshold of exposure below which no biologically significant effect will be produced. There is ongoing debate about whether there are true biological thresholds in the dose-response curves.

This method involves use of uncertainty factors to allow for interspecies differences and human variability - it moves the dose-response curve from the response for a group of experimental animals down to the curve for sensitive humans. Both factors are 10 giving an overall factor of 100, which has been used internationally now for 40 years.

This approach will be included in the study because uncertainty factors applied to NOAEL is the standard approach adopted by all agencies undertaking risk assessment.

\subsection{Non-threshold methods}

For some hazards it is considered that there may be no threshold for the mode of action, and therefore a level of exposure without significant adverse effects cannot be determined. Attempts to provide estimates of exposure associated to risks in the region of, for example, 1 in $10^{6}$ are usually made.

This approach normally requires extrapolation of the dose-response relationship over at least four orders of magnitude. According to the extrapolation used different methods exist. Species differences can be taken into account. Though there is no clear consensus and harmonisation on the various approaches which have been used internationally, this approach is included because of the nature of the problem, which suggests that threshold for endocrine disrupting compounds may not exist.

\subsection{Probabilistic risk assessment}

In threshold methods the acceptable daily intake (ADI) is calculated in the following way

$$
A D I=\frac{N O A E L}{U F_{1} \times U F_{2} \times \ldots}
$$


where NOAEL is the No Observable Adverse Effect Level and $U_{F}$ is the $i^{\text {th }}$ uncertainty factors. This approach provides a single value, the uncertainty of which cannot be quantified.

In the Probabilistic Risk Assessment (PRA), the aim is to derive a range of values that are plausible, given the uncertainty in our general scientific knowledge, as well as the available data. An important advantage of the PRA is that it allows for the estimation of possible health effects given the actual exposure in the population. PRA has not received an official status yet, but is being increasingly recognized.

\section{Concluding remarks}

The main objective of F\&F project is to determine the risks from pharmaceutical products that may end up in the food chain with an effect on human fecundity. Some studies indicate that there is some decrease in fecundity in certain areas of Europe, but it is not clear whether this is due to change in lifestyle or due to some environmental factors and/or presence of certain PPs in food.

The risk assessment (RA) part takes very important role in the F\&F project since the RA is ultimately going to produce some estimate of the associated risks to human fecundity due to presence of PPs in food.

\section{Acknowledgement}

The financial support by the European Commission through the FP6 - Food Quality and Safety programme, Contract No. FOOD-CT-2004-513953, is gratefully acknowledged.

\section{References}

[1] Luijten M, Tomenko V, Popov V, Piersma AH (2005). A scenariospecific prioritization list of chemicals bearing a high potential of affecting human fecundity, Deliverable number D1.1, F\&F, Contract No. FOOD-CT-2004513953.

[2] Archand-Hoy, L.D., Nimrod, A.C. and Benson, W.H. (1998) Endocrinemodulating substances in the environment: estrogenic effects of pharmaceutical products. International Journal of Toxicology 17, 139-158.

[3] Webb, S. (2000) Risk assessment for pharmaceuticals. In: Proceedings of International Seminar on Pharmaceuticals in the Environment. March 9th 2000, Brussels, Tecnological Institute.

[4] Archand-Hoy, L.D., Nimrod, A.C. and Benson, W.H. (1998) Endocrinemodulating substances in the environment: estrogenic effects of pharmaceutical products. International Journal of Toxicology 17, $139-158$. 
[5] Ternes, T.A., M. Stumpf, J. Mueller, K. Haberer, R.D. Wilken, and M. Servos. (1999). Behavior and occurrence of estrogens in municipal sewage treatment plants - I. Investigations in Germany, Canada and Brazil. Sci. Total Environ. 225:81-90.

[6] Larsson, D.G.J., Adolfsson-Erici, M., Parkkonen, J., Pettersson, M., Berg, A.H., Olsson, P.-E., Forlin, L. (1999) Ethinyloestradiol — an undesired fish contraceptive? Aquatic Toxicology, 45, 91-97.

[7] Aherne, G.W., Briggs, R. (1989) The relevance of the presence of certain synthetic steroids in the aquatic environment. $J$ Pharm Pharmacol 41, 735-736.

[8] Hohenblum, P., Gans, O., Moche, W., Scharf, S., Lorbeer, G. (2004) Monitoring of selected estrogenic hormones and industrial chemicals in groundwaters and surface waters in Austria, Science of the Total Environment, 333, 185- 193.

[9] Kuhnz W, Heuner A, Humpel M, Seifert W, Michaelis K. (1997) In vivo conversion of norethisterone and norethisterone acetate to ethinyl etradiol in postmenopausal women. Contraception,56, 379-385.

[10] Ternes, T.A., Kreckel, P., Mueller, J. (1999) Behavior and occurrence of estrogens in municipal sewage treatment plants - II. Aerobic batch experiments with activated sludge. Sci Total Environ, 225, 91-99.

[11] Jurgens, M.D., Holthaus, K.I.E., Johnson, A.C., Smith, J.J.L., Hetheridge, M., Williams, R.J., 2002. The potential for estradiol and ethinylestradiol degradation in English rivers. Environ. Toxicol. Chem. 21 (3), 480-488.

[12] Hartmann, S., Lacorn, M., and Steinhart, H. 1998. Natural occurrence of steroid hormones in food. Food Chemistry, 62/1, pp. 7-20. 